OPEN ACCESS

Edited by:

Heike Wulf,

University of California, Davis,

United States

Reviewed by:

Chai K. Lim,

Macquarie University, Australia

Gayathri Sundaram,

St. Vincent's Hospital, Australia

${ }^{*}$ Correspondence:

Gabriela D. Colpo

gabicolpo@gmail.com

Antonio L. Teixeira

antonio.I.teixeira@uth.tmc.edu

Specialty section

This article was submitted to

Stroke,

a section of the journal

Frontiers in Neurology

Received: 26 March 2019

Accepted: 03 July 2019

Published: 19 July 2019

Citation:

Colpo GD, Venna VR, McCullough LD and Teixeira AL (2019) Systematic

Review on the Involvement of the

Kynurenine Pathway in Stroke:

Pre-clinical and Clinical Evidence.

Front. Neurol. 10:778.

doi: 10.3389/fneur.2019.00778

\section{Systematic Review on the} Involvement of the Kynurenine Pathway in Stroke: Pre-clinical and Clinical Evidence

\author{
Gabriela D. Colpo ${ }^{*}$, Venugopal R. Venna ${ }^{2}$, Louise D. McCullough ${ }^{2}$ and \\ Antonio L. Teixeira ${ }^{1 *}$
}

${ }^{1}$ Neuropsychiatry Program, Department of Psychiatry and Behavioral Sciences, University of Texas Health Science Center at Houston, Houston, TX, United States, ${ }^{2}$ BRAINS Lab, Department of Neurology, University of Texas Health Science Center at Houston, Houston, TX, United States

Background: Stroke is the second leading cause of death after ischemic heart disease and the third leading cause of disability-adjusted life-years lost worldwide. There is a great need for developing more effective strategies to treat stroke and its resulting impairments. Among several neuroprotective strategies tested so far, the kynurenine pathway (KP) seems to be promising, but the evidence is still sparse.

Methods: Here, we performed a systematic review of preclinical and clinical studies evaluating the involvement of KP in stroke. We searched for the keywords: ("kynurenine" or "kynurenic acid" or "quinolinic acid") AND ("ischemia" or "stroke" or "occlusion) in the electronic databases PubMed, Scopus, and Embase. A total of 1,130 papers was initially retrieved.

Results: After careful screening, forty-five studies were included in this systematic review, being 39 pre-clinical and six clinical studies. Despite different experimental models of cerebral ischemia, the results are concordant in implicating the KP in the pathophysiology of stroke. Preclinical evidence also suggests that treatment with kynurenine and $\mathrm{KMO}$ inhibitors decrease infarct size and improve behavioral and cognitive outcomes. Few studies have investigated the KP in human stroke, and results are consistent with the experimental findings that the $\mathrm{KP}$ is activated after stroke.

Conclusion: Well-designed preclinical studies addressing the expression of KP enzymes and metabolites in specific cell types and their potential effects at cellular levels alongside more clinical studies are warranted to confirm the translational potential of this pathway as a pharmacological target for stroke and related complications.

\footnotetext{
Keywords: stroke, kynurenine pathway, kynurenic acid, quinolinic acid, KMO, IDO
} 


\section{INTRODUCTION}

Stroke is clinically defined by the sudden onset of focal neurological symptoms (motor, sensory, cognitive) due to ischemia or hemorrhage in the brain. It is the second leading cause of death after ischemic heart disease and the third leading cause of disability-adjusted life-years lost worldwide (1). In the last two decades, there has been significant advance in the acute management of stroke, including the establishment of dedicated stroke inpatient units and the use of thrombolysis for eligible patients with ischemic stroke. Despite this progress, stroke-related deaths and morbidity remain a major health problem with personal and societal implications.

To address the great need of advancing stroke management, several mechanisms implicated in the pathophysiology of stroke, such as mitochondria dysfunction, glutamate-induced excitotoxicity, neuroinflammation, oxidative stress, among others, have been investigated as therapeutic targets. Among putative candidates, the kynurenine pathway (KP) received attention in the 1990's with a renewed interest recently on the wave of inflammatory-centric perspective of central nervous system diseases, including stroke (2).

The KP is the major route of tryptophan (TRP) catabolism in mammals. TRP is an essential amino acid used in the biosynthesis of proteins, being also a precursor of several bioactive molecules, such as serotonin and melatonin. Around $90 \%$ of TRP is metabolized by tryptophan 2,3-dioxygenase (TDO) into kynurenine (KYN) in the liver, with a much lower contribution of extra-hepatic KP on TRP degradation (5-10\%) (3). TDO is liver specific, but two TDO variants been identified in mouse brain structures during development (4).

In extrahepatic tissues, especially cells of the immune and central nervous systems, the KP is initiated by the degradation of TRP by indoleamine 2, 3-dioxygenase 1 (IDO), the rate limiting enzyme of the pathway. This enzyme is potently upregulated by pro-inflammatory stimuli (2). After this step, the KP branches into two major pathways-one implicated in neuroprotection, the other in neurotoxicity-that are segregated across cell types (Figure 1). Under physiological conditions, the neuroprotective branch is more active as most of kynurenine in the brain is metabolized into kynurenic acid, a NMDA and $\alpha 7$ nicotinic acetylcholine receptor antagonist, through the action of kynurenine aminotransferases (KATs) expressed mainly in astrocytes (5). Under inflammatory conditions, the metabolism is shifted through kynurenine-3-monooxygenase (KMO) to produce 3-hydroxykynurenine and other toxic metabolites,

Abbreviations: 3-HK, 3-hydroxykynurenine; AhR, aryl hydrocarbon receptor; BCCAO, Bilateral Common Carotid Artery Occlusion; Ccbf, Corticocerebral blood flow; CNS, Central nervous system; GFAP, Glial fibrillary acidic protein; ICAM-1, Intercellular Adhesion Molecule 1; IDO1, indoleamine 2,3-dioxygenase 1; IDO2, indoleamine 2,3-dioxygenase 2; IFN- $\gamma$, Interferon gamma; IL-10, interleukin-10; IPC, Ischemia preconditioning; KATs, Kynurenine aminotransferase; KMO, Kynurenine 3-monooxygenase; KYNA, Kynurenic acid; L-KYN, Kynurenine; LTP, Long-term potentiation; MCAO, Middle Cerebral Artery Occlusion; NMDA, N-methyl-D-aspartate; OGD, Glucose deprivation; PIT, Photochemically induced thrombosis; QUIN, Quinolinic acid; 4VO, Four-vessel occlusion; TDO, Tryptophan-2,3-dioxygenase; TNF- $\alpha$, Tumor Necrosis Factor Alpha; TRP, Tryptophan; WB, Western blot.

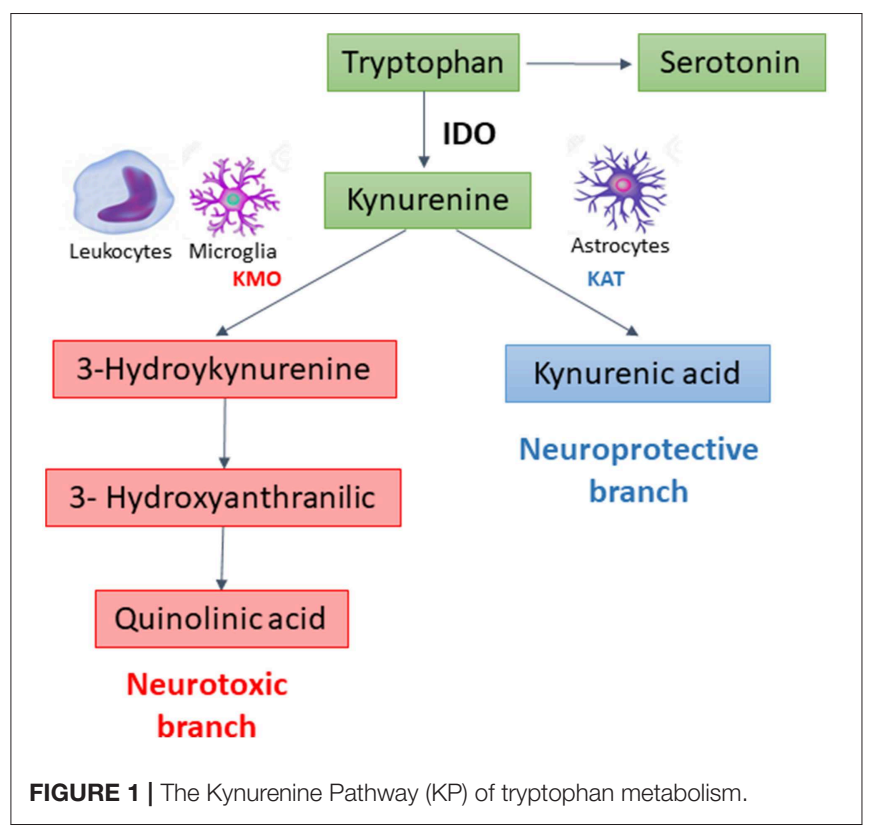

including quinolinic acid, a NMDA receptor agonist and an oxidative stressor $(3,6)$. $\mathrm{KMO}$ is primarily expressed in microglia, the resident immune cells in the brain, and is also expressed at high levels in peripheral immune cells such as monocytes/macrophages (7).

Besides kynurenic acid (KYNA) and quinolinic acid (QUIN), the KP produces several other biologically active metabolites, including the redox cofactors oxidized nicotinamide adenine dinucleotide (NAD+). NAD is a common mediators of various biological processes, including energy metabolism, mitochondrial functions, calcium homeostasis, antioxidation/generation of oxidative stress (8). During cerebral ischemia, NAD is rapidly depleted, and increasing NAD has been proposed as a potential therapeutic strategy against stroke (9). Both NADPH and NAD+ have been reported to display potent neuroprotective effects in ischemia-related neuronal injury (10).

After an ischemic stroke, a series of inflammatory events takes place, leading to activation of resident microglia and mobilization of peripheral leukocytes with their subsequent infiltration into the injured side (11). Infiltrating leukocytes release inflammatory mediators, amplifying the intrinsic brain inflammatory response. Theoretically, these mechanisms could lead to activation of the KP, mainly its "neurotoxic" branch, contributing to neuronal damage and, ultimately, to clinical outcome (3). There is some pre-clinical and clinical evidence indicating activation of KP in stroke. Besides playing a pathophysiological role, KP metabolites have been tested as pharmacological agents to prevent and/or minimize stroke-related brain damage.

In this systematic review, our main objective was to summarize the evidence on the involvement of KP in both experimental models of brain ischemia and human patients with stroke. 


\section{METHODS}

\section{Systematic Search}

We undertook a comprehensive systematic search to identify all published studies evaluating KP in clinical studies and animal models of brain ischemia or stroke using the electronic databases PubMed, Scopus, and Embase. We conducted the search based on the keywords: ("kynurenine" or "kynurenic acid" or "quinolinic acid") AND ("ischemia" or "stroke" or "occlusion"). Studies published through November 2018 were included.

A total of 1,130 papers was retrieved after removing duplicate manuscripts. Reference management software (EndNote X7 for Windows from Thomson Reuters, 2013) was used for screening purposes. The systematic review was performed in accordance with the Preferred Reporting Items for Systematic Reviews and Meta-Analysis (PRISMA) statement (12) as depicted in Figure 2.

\section{Eligibility Criteria, Data Extraction, and Quality Assessment}

Studies were considered eligible to be included in the review if they met the following criteria: (1) animal model of stroke or brain ischemia and evaluation of KP metabolites or enzymes; (2) study with stroke patients and evaluation of KP metabolites or enzymes; (3) available in English; and (4) original data (not a review). The exclusion criteria were: (1) review manuscripts, (2) in vitro studies, (3) only abstracts and (4) non-relevant studies. During December 2018 and January 2019, one author (GDC) screened the titles and abstracts of all articles. Full texts were obtained for all articles that met the inclusion criteria $(n=50)$. Full-texts of the eligible studies were independently screened by two authors (GDC and ALT) who identified aims, methods, results and conclusions, extracting the respective information. Five papers were excluded in this phase.

To assess the risk of bias of the pre-clinical studies included in this systematic review, we used the SYRCLE's RoB tool (13). This tool evaluates specific features that might be source of bias in animal studies such as group allocation, if animals were randomly housed during the experiment, blinded investigators, animals selected at random for outcome assessment.

\section{RESULTS}

In the end of the screening process, 45 studies met the inclusion criteria and they were included in this systematic review. Among them, 39 studies involved animal models of stroke/ brain ischemia and six were clinical studies comprising patients with

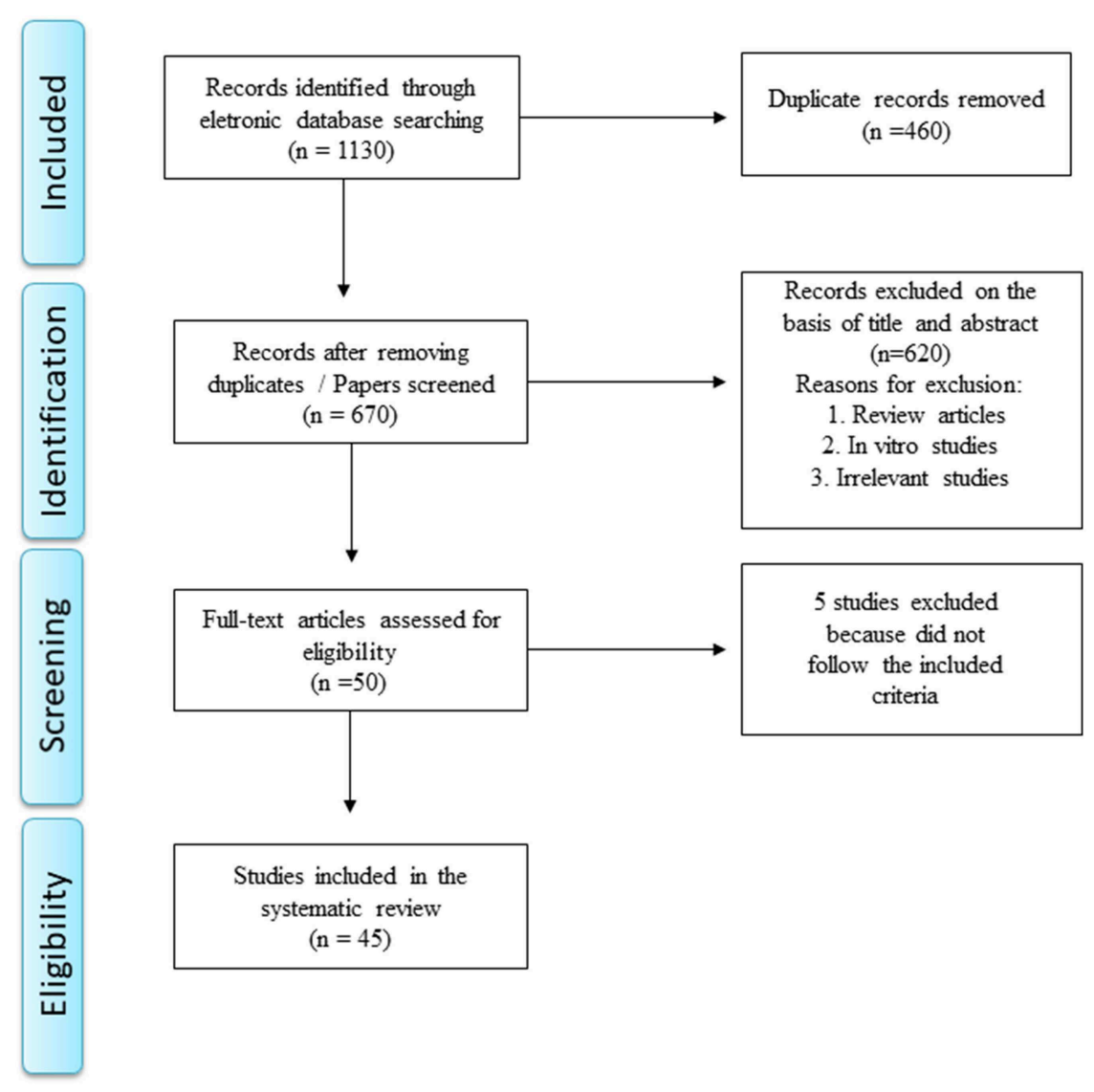

FIGURE 2 | Flow diagram. 
stroke. These studies are summarized in Tables 1, 2, while the main results are summarized in Table 3.

\section{Pre-clinical Studies}

Regarding the quality assessment of the pre-clinical studies, only five papers reported that randomization was carried out, but without stating the method of group allocation. All studies reported that age and/or weight were similar among groups. None of the studies described whether animals were randomly selected for outcome assessment.

The type of animal model varied among the studies. More than 25 studies used rats while the second most used animal was the Mongolian gerbil followed by mice. Two studies used rabbits. Only six studies used females: one study with rats and five studies with gerbils, but none specifically examined the effects by sex. No study assessed aged animals.

The main aim of pre-clinical investigation was to assess the neuroprotective effects of KP metabolites (kynurenine or kynurenic acid) and/or KMO inhibitors (mNBA and Ro 61-8048) in different models of ischemia. Overall, studies with kynurenine and KMO inhibitors showed positive results, i.e., decrease in the infarct size and increased survival with treatment.

Gigler et al. (40) showed that intraperitoneal (i.p.) administration of kynurenine before stroke by middle cerebral artery occlusion reduced infarct size and hippocampal CA1 pyramidal cell loss in adult male mice. This same strategy also prevented post-stroke behavioral changes in gerbils in the bilateral carotid occlusion model (40). Pre- and post-treatment with kynurenine decreased cortical neuron damage in adult rats subjected to four-vessel occlusion (41).

Cozzi et al. evaluated the neuroprotective effects of KMO inhibitors. The KMO inhibitors mNBA and Ro 61-8048 were tested as neuroprotective strategies against ischemia induced by bilateral carotid occlusion in male Mongolian gerbils or middle cerebral artery occlusion in male rats. In gerbils, the percentage of lesioned pyramidal neurons in the hippocampal $\mathrm{CAl}$ region decreased significantly from around $90 \%$ in vehicletreated animals to $7 \%$ after mNBA ( $400 \mathrm{mg} / \mathrm{kg}$ i.p., three times at 1,30, and $180 \mathrm{~min}$ after occlusion) or 10\% after Ro61-8048 ( $40 \mathrm{mg} / \mathrm{kg}$ i.p., three times at 1,30, and $360 \mathrm{~min}$ after occlusion). A major reduction in infarct volumes was also found in rats after middle cerebral artery occlusion treated with mNBA or Ro 61-8048 administrated $30 \mathrm{~min}$ after the occlusion (31).

The results are mixed regarding the effects of kynurenic acid and analogs. Kynurenic acid did not show any protective effects in two studies $(17,48)$. Roussel et al. for instance, showed that kynurenic acid did not modify neither infarct volume or the neurological score $48 \mathrm{~h}$ after middle cerebral artery occlusion in adult male rats (17). Conversely, Gellert et al. (45) showed that administration of a kynurenic acid analog (2-(2-N,N-dimethylaminoethylamine-1-carbonyl)-1H-quinolin4-one hydrochloride) either before or after ischemia reduced hippocampal CA1 pyramidal cell loss and preserved long-term potentiation expression at the Schaffer collateral-CA1 synapses in adult male rats in a four-vessel occlusion model. Other study showed that pretreatment with systemic administration of KYN (i.p.) reduced infarct size in mice and hippocampal
CA1 pyramidal cell loss in gerbils, preventing post-stroke behavioral changes as hypermotility and decreased spontaneous alternation (40).

Pre-clinical studies also addressed the pathophysiological involvement of the $\mathrm{KP}$ after stroke, mainly focusing on the expression of the enzyme IDO and the metabolite kynurenic acid. Hoshi et al. showed by mRNA and immunohistochemistry increased expression of IDO in hippocampal CA1 area of adult male mice $72 \mathrm{~h}$ after global cerebral ischemia and that IDO is co-localized with Neu-N, a neuronal marker (43). Increased IDO expression was also observed in endothelial cells from cerebral arterioles $24 \mathrm{~h}$ post-middle cerebral artery occlusion, with increased plasma kynurenine/tryptophan ratio, an index of IDO activity, in adult male mice (46). Increased IDO activity and quinolinic acid concentrations in hippocampus, striatum, cortex, and thalamus, but not in cerebellum, were observed 4 days after $10 \mathrm{~min}$ of ischemia by bilateral common carotid artery occlusion in female Mongolian gerbils. Increased activity of kynureninase, kynurenine 3-hydroxylase, and 3hydroxyanthranilate-3, 4-dioxygenase was also observed in hippocampus but not in cerebellum on day 4 (23). Most studies found increased expression of kynurenic acid in the stroke area. Kynurenic acid expression was increased in the infarcted areas from day 2 to 21 post-ischemia caused by single transient middle cerebral artery occlusion in adult male rats (52).

\section{Clinical Studies}

Only six clinical studies were identified. In these studies, patients were recruited within the first hours of the stroke, and different molecules such as tryptophan, kynurenine and kynurenic acid were measured in parallel with clinical parameters. Only two studies compared stroke patients with healthy controls, while the remaining four investigated whether KP-related molecules could be used as biomarkers of prognosis or clinical outcomes.

Ximing et al. studied 81 patients with ischemic stroke and 35 healthy controls, and reported a decrease in serum levels of tryptophan, kynurenic acid and KAT activity (calculated by kynurenic acid/kynurenine ratio) in the stroke group. Conversely, the levels of highly sensitive $\mathrm{C}$ reactive protein (hsCRP), a marker of inflammation, and IDO activity, as assessed by the kynurenine/tryptophan ratio, were significantly higher in the stroke group compared to controls. IDO activity showed a positive correlation with hsCRP, while hsCRP levels and IDO activity were positively correlated with the severity of the stroke as estimated by the NIH Stroke Scale both at admission and 3 weeks post-stroke (57). The other study investigated whether the $\mathrm{KP}$ activation correlated with infarct volume in 50 patients with acute stroke and 35 healthy control subjects (53). Tryptophan levels were significantly lower in stroke patients compared to controls at three time points: 1, 7, and 14 days after stroke. Kynurenine levels were increased in patients compared with controls on the first day post-stroke but not at subsequent time points. These results suggest that increased tryptophan catabolism is initiated immediately after the ischemic event, likely triggered by the inflammatory response and oxidative stress, with a major change in 3-hydroxyanthranilic acid levels that strongly correlated with infarct volume (53). Of note, 
TABLE 1 | Animal model studies with stroke/ischemia and kynurenine pathway.

\begin{tabular}{|c|c|c|}
\hline References & Animal model & Results \\
\hline Simon et al. (14) & $\begin{array}{l}\text { Kynurenate in anoxic ischemic } \\
\text { brain injury in neonatal rats }\end{array}$ & $\begin{array}{l}\text { These results show a marked attenuation in brain } \\
\text { water in ischemic hemispheres of } \\
\text { kynurenate-treated } 7 \text {-day-old animals that is } \\
\text { greatest at } 24 \mathrm{~h} \text {. In regard to neuronal } \\
\text { proliferation and migration, the gestational age of } \\
7 \text { days in rats is compatible with that of newborn } \\
\text { humans. }\end{array}$ \\
\hline Germano et al. (15) & $\begin{array}{l}\text { KYNA in focal cerebral ischemia } \\
\text { in adult male Sprague-Dawley } \\
\text { rats. }\end{array}$ & $\begin{array}{l}\text { Pre-ischemia but not } 1 \mathrm{~h} \text { post-ischemia treatment } \\
\text { with kynurenate attenuated infarction size and } \\
\text { improved neurological outcome at } 24 \mathrm{~h} \text { after } \\
\text { injury. }\end{array}$ \\
\hline Andine et al. (16) & KYNA in hypoxic-ischemia in & KYNA reduced weight of lesioned hemisphere. \\
\hline
\end{tabular}

Andine et al. (16)

Roussel et al. (17) neonatal rats.

Kynurenate in male Wistar-Kyoto (spontaneously hypertensive) SHRs rats with cerebral occlusion

Heyes and Nowak (18)

Roucher et al. (19)

Katayama et al. (20)

Lekieffre et al. (21)

Nozaki and Beal (22)
Metabolism of the endogenous excitotoxin, QUIN in ischemia in female Mongolian gerbils

Kynurenate in ischemia in male Wistar rat.

KYNA administered in transient ischemia in gerbils and Sprague-Dawley rats.

KYNA in male Wistar rats with ischemia.

KYNA in hypoxia-ischemia in 7-day-old rats
Kynurenate did not significantly modify either the infarct volume, measured $48 \mathrm{~h}$ after occlusion, or the neurological score.

High brain TRP levels, and increased 5-hydroxyindoleacetic acid, occurred during the first hours of recirculation, but regional brain QUIN concentrations decrease during the first $24 \mathrm{~h}$ after the ischemia. Increases in QUIN concentrations occurred in striatum and hippocampus at 2 days of recirculation after ischemia. After, 4 and 7 days, QUIN increases in all regions.

Kynurenate had no effect on cerebral metabolism before ischemia. During a $30 \mathrm{~min}$ ischemia, kynurenate protected against the decrease in phosphocreatine and the increase in phosphate whereas there was no difference in the decrease in intracellular $\mathrm{pH}$ and ATP. The recovery of PCr, $\mathrm{Pi}$, and $\mathrm{pHi}$ to control levels during recirculation was faster in the treated group than in the reference group, whereas the time course of ATP recovery was similar in both groups.

KYNA attenuates the increase in extracellular concentration of lactate during ischemia and attenuates death of hippocampal CA1 pyramidal cells after 5-min transient ischemia in gerbils.

KYNA decreased glutamate release during ischemia but had no effect on the hippocampal lesion. Some protection was observed in the cortex and in the striatum.

L-KYN $1 \mathrm{~h}$ before the hypoxia-ischemia showed a dose-dependent neuroprotective effect, with complete protection at a dose of $300 \mathrm{mg} / \mathrm{kg}$. The induction of c-fos immunoreactivity in cerebral cortex was also blocked by this dose of L-KYN. Probenecid alone had moderate neuroprotective effects, while a combination of a low dose of probenecid with doses of 50-200 mg kg-1 of L-KYN showed significant dose-dependent neuroprotection. KYN dose-dependently protected against NMDA neurotoxicity in 7-day-old rats.
Conclusion

The results suggest that kynurenate treatment attenuated brain edema immediately after and $24 \mathrm{~h}$ following anoxia-ischemia.

The results suggest the role of excitatory neurotransmission in acute neuronal injury and supports pharmacological inhibition of cell excitation as a potential therapy for stroke.

The results suggest that excitatory amino acids are involved in the development of post ischemic damage in the immature brain.

The results suggest that the absence of a neuroprotective effect of kynurenate, which contrasts with results in normotensive rats, is possibly due to impaired collateral circulation in spontaneously hypertensive rats.

The results do not support a role for increased QUIN concentrations in early excitotoxicity-dependent neuronal damage. The role of the delayed increases in brain QUIN in the progression of post ischemic injury remains to be established.

The results suggest that kynurenate protects against neuronal loss by mechanisms other than metabolic protection.

The results suggest that KYNA attenuates the death of hippocampal cells after ischemia in gerbils. The protective effect of KYN may be attributable, in part, to inhibition of lactate accumulation.

The results suggest that the extracellular accumulation of glutamate during forebrain ischemia does not play a major role in hippocampus damage.

The results show for the first time that pharmacological manipulation of endogenous concentrations of KYNA exerts neuroprotective effects. 
TABLE 1 | Continued

\begin{tabular}{|c|c|c|c|}
\hline References & Animal model & Results & Conclusion \\
\hline Saito et al. (23) & $\begin{array}{l}\text { IDO activity and QUIN } \\
\text { concentrations in transient } \\
\text { ischemia in the female Mongolian }\end{array}$ & $\begin{array}{l}\text { Increases in IDO activity and QUIN } \\
\text { concentrations were found } 4 \text { days after ischemia, } \\
\text { with responses in hippocampus > striatum }\end{array}$ & $\begin{array}{l}\text { The results suggest that kynurenine } \\
\text { 3-hydroxylase may have an important role in } \\
\text { determining the flux of kynurenine in brain. }\end{array}$ \\
\hline
\end{tabular}

Saito et al. (24) KYN and QUIN in ischemic brain in female Mongolian gerbils and after systemic administration of pokeweed mitogen

3-hydroxylase activity in female Mongolian gerbils and male Sprague-Dawley rats, male macaques and humans.

Indole-pyruvic acid (IPA) in male Sprague-Dawley rats.

KYNA in ischemia in male Wistar rats

Ghribi et al. (27)
$>$ cerebral cortex > thalamus. IDO and QUIN concentrations were unchanged in the cerebellum of post ischemic gerbils. Marked increases in the activity of kynureninase, kynurenine 3-hydroxylase, and 3-hydroxyanthranilate-3, 4-dioxygenase were also detected in hippocampus but not in cerebellum on day 4 of recirculation. Accumulation of QUIN was demonstrated in cerebellum and hippocampus of control gerbils following an intracisternal injection of 3-HAA, which verifies the availability of precursor to both regions when administered intracisternally.

One day after ischemia, kynureninase and 3-hydroxy-3,4-dioxygenase were increased in the hippocampus, but local QUIN levels and the activities of the IDO and kynurenine-3-hydroxylase were unchanged. By days 2 and 4 after ischemia, the activities of all these enzymes in the hippocampus as well as QUIN levels were increased. KAT activity in the hippocampus was unchanged on days 1 and 2 after ischemia but was decreased on day 4 , at a time when local KYNA levels were unchanged. Systemic administration of pokeweed mitogen increased indoleamine-2,3-dioxygenase and kynureninase activities in the brain without changes in kynurenine-3-hydroxylase or 3-hydroxyanthranilate-3,4-dioxygenase activities. Gerbil brain activities ranged from 20 to 50 $\mathrm{nmol} / \mathrm{g} / \mathrm{h}$, while kynurenine 3-hydroxylase activities in other species were one order of magnitude lower. Kynurenine 3-hydroxylase was also detected in lung, kidney, spleen, intestine, and liver of gerbils, with activities larger than in brain.

Analysis showed a protective effect of IPA treatment on striatal ischemic lesions. Increased neuronal loss, regional atrophy and glial fibrillary acidic protein immunoreactivity were observed in the hippocampal formation, especially the CA3 field, of IPA-treated rats when compared with vehicle-treated ischemic rats. The treatment with IPA did not produce any improving effects in a test assessing short-term impairments after transient ischemia. No effects of IPA on performance in water T-maze studied at 7 and 14 days post-ischemia.
KYNA markedly depressed the ischemia-induced increase in glutamate and aspartate concentrations.
The results support a role for macrophage infiltration and increased activity and colocalization of IDO, kynurenine-3-hydroxylase and kynureninase in conveying the ability of brain tissue to convert L-tryptophan to QUIN in neuroinflammation.

The results suggest that delayed increase in KYN pathway metabolism occurs in different brain regions following transient ischemia in the gerbils. These changes are marked in regions that show the most extensive damage and inflammatory responses and are consistent with a localization of the induction of KYN pathway to reactive macrophages.

The results suggest that IPA does not have neuroprotective effects against ischemia in the hippocampus.
The results indicate that during ischemia, local glutamate receptors play a major role in glutamate and aspartate accumulation in the striatum. Ischemia increase in extracellular concentration of excitatory amino acids may be due in part to a positive glutamatergic feedback loop via activation of NMDA and/or non-NMDA receptors. 
TABLE 1 | Continued

\begin{tabular}{|c|c|c|}
\hline References & Animal model & Results \\
\hline Saito et al. (28) & $\begin{array}{l}\text { 3-hydroxy-anthranilate- } \\
\text { 3,4-dioxygenase and } \\
\text { dexamethasone in female } \\
\text { Mongolian gerbils with ischemia. }\end{array}$ & $\begin{array}{l}\text { 3-hydroxy-anthranilate-3,4-dioxygenase } \\
\text { significantly reduced the accumulation of QUIN in } \\
\text { all brain regions in response to cerebral ischemia. }\end{array}$ \\
\hline Heyes et al. (29) & $\begin{array}{l}\text { Quinolinate responses to } \\
\text { systemic and brain immune } \\
\text { activation in gerbils, } \\
\text { Sprague-Dawley and Wistar rats }\end{array}$ & $\begin{array}{l}\text { Global cerebral ischemia in gerbils, but not rats, } \\
\text { increased hippocampus } \\
\text { indoleamine-2,3-dioxygenase activity and } \\
\text { quinolinate levels } 4 \text { days post injury. In rats, small } \\
\text { increases in quinolinate concentrations occurred } \\
\text { in infarcted regions on days } 1,3 \text {, and } 7 \text {, although } \\
\text { concentrations remained below serum values. }\end{array}$ \\
\hline Baratte et al. (30) & $\begin{array}{l}\text { QUIN immunoreactivity in adult } \\
\text { male mongolian gerbils with } \\
\text { transient global ischemia. }\end{array}$ & $\begin{array}{l}\text { Neurodegeneration was evident in hippocampus } \\
4,7 \text {, and } 14 \text { days after ischemia. QUIN positive } \\
\text { cells, with microglia-like morphology, appeared in } \\
\text { the subiculum and in the CA1, } 4 \text { days after } \\
\text { ischemia. At } 7 \text { days post-ischemia they extended } \\
\text { to the whole CA1, disappearing at } 14 \text { days. }\end{array}$ \\
\hline
\end{tabular}

Cozzi et al. (31) mNBA and Ro 61-8048 in Mongolian gerbils and male Sprague-Dawley rats.

Phillis et al. (32)

KYNA in ischemic cerebral cortex in Male Sprague-Dawley rats.

Salvati et al. (33)

KYNA (400-1,600 mg/kg) or vehicle were administered i.p. $15 \mathrm{~min}$ before $5 \mathrm{~min}$ bilateral carotid occlusion in adult male Mongolian gerbils.

The percentage of lesioned pyramidal neurones decreased in gerbils. Also, significant reduction in infarct volumes in rats with the inhibition of kynurenine hydroxylase.

KYNA, administered topically onto the cortex in artificial cerebrospinal fluid using bilateral cortical cups, failed to attenuate ischemia-evoked release of aspartate, glutamate, phosphoethanolamine, taurine and at $1 \mathrm{mM}$ it depressed GABA release.

Seven days after reperfusion, ischemia-induced hippocampal nerve cell loss was lower in KYNA-treated gerbils. Treatment with 1,000 $\mathrm{mg} / \mathrm{kg}$ produced brain KYNA concentrations that were dramatically elevated, as measured in a separate group of transcardially-perfused gerbils. Cerebral KYNA concentrations tended to return to basal values $2 \mathrm{~h}$ after reperfusion.

Edwards et al. (34) Concentrations of glutamate and kynurenate after diclofenac or noxious stimulation in Male Sprague Dawley rats

The glutamate concentrations were lower in the ischemia group when compared with control and diclofenac-treated groups. Diclofenac-treated groups found significant increases in kynurenate concentrations in the diencephalon and lumbo-sacral regions of the CNS.

Moroni et al. (35)

Kynurenine hydroxylase inhibitors in gerbils, rats and organotypic rat culture.

Luchowska et al. (36)

Levels of KYNA after global ischemia in Mongolian gerbils
In organotypic hippocampal slice cultures deprived of oxygen and glucose, these inhibitors reduced neuronal damage. In gerbils the administration of mNBA or Ro 61-8048 decreased the percentage of damaged pyramidal neurons in the hippocampal CA1 region. In rats Ro 61-8048 administration reduced the infarct volume.

The level of KYNA in CA1 area was not altered 24 and $72 \mathrm{~h}$ following transient global. Similarly, the activities of KATs in CA1 area were not changed.
Conclusion

The results suggest that increased QUIN concentration in specific brain regions is linked with enhanced activities of IDO and other kynurenine pathway enzymes.

The results demonstrate a limited capacity of rats to elevate in brain and blood QUIN levels in response to immune activation is attributable to increases in local indoleamine-2,3-dioxygenase activity and a low capacity of microglia, astrocytes, and macrophages to convert L-tryptophan to quinolinate.

The results suggest that microglia-like cells infiltrating the degenerating areas of the hippocampus represent the major source of QUIN following transient ischemia in the gerbil. Thus, in situ production of QUIN in vulnerable brain regions may contribute to the pathophysiological mechanisms of delayed brain injury.

The results suggest that inhibition of kynurenine hydroxylase could be a new avenue to reduce neuronal loss in brain ischemia.

The results suggest that ischemia-evoked amino acid release is not a significant consequence of the activation of ionotropic receptors by glutamate.

The results indicate that KYNA has a neuroprotective effect in a model of forebrain ischemia, compatible with in vitro affinity of this molecule for ionotropic glutamate receptors.

The results suggest that noxious stimulation from tail ischemia appeared to be associated with increased release of glutamate. NSAIDs increase kynurenate concentrations in the spinal cord and diencephalon. Antagonism by kynurenate of glutamate effects at NMDA receptors may contribute to the antinociceptive effects of NSAIDs

The results demonstrate that ischemic neuronal damage can be decreased by inhibiting kynurenine hydroxylase.

The results indicate that KYNA production is preserved in CA1 area of gerbil hippocampus during early stages after ischemic insult. 
TABLE 1 | Continued

\begin{tabular}{|c|c|c|c|}
\hline References & Animal model & Results & Conclusion \\
\hline Moroni et al. (37) & $\begin{array}{l}\mathrm{KMO} \text { in gerbils and organotypic } \\
\text { rat hippocampal slice }\end{array}$ & $\begin{array}{l}\text { In gerbils, completely prevented the increase in } \\
\text { Glu output induced by transient occlusion of the }\end{array}$ & $\begin{array}{l}\text { The results suggest that } \mathrm{KMO} \text { inhibitors prevent } \\
\text { neuronal death by decreasing } 3-\mathrm{HK} \text { synthesis. }\end{array}$ \\
\hline
\end{tabular}

Sas et al. (38)

Abo et al. (39)

Gigler et al. (40)

Robotka et al. (41)

Sas et al. (42)

Hoshi et al. (43)

Sas (44)

Gellert et al. (45)

Jackman et al. (46)
L-KYN effects in New Zealand white rabbits with ischemic.

KYNA in female Fischer rats

MCAO in male NMRI mice + BCCAO in gerbils

L-KYN in ischemic in adult Wistar rats

t4VO in adult male Wistar rats

tBCCAO in adult male C57BL/6J mice

KYN in adult male Wistar rats with ischemia and L-KYN and New-Zealand white rabbits with ischemic condition

$4 \mathrm{VO}$ in adult male Wistar rats

tMCAO in adult male C57BL/6 mice carotids. In rat hippocampal slices exposed for

30 min to OGD, KMO inhibitors reduced post-ischemic neuronal death and increased KYNA concentrations in the incubation medium. On the contrary, 3-HK added to slices exposed to OGD in the presence of KMO inhibitors completely prevented the neuroprotective effects of the inhibitors.

$\mathrm{L}-\mathrm{KYN}$ produced an increase in the normal cCBF. The cCBF-improving effect of L-KYN was immediate and highly in rabbits with carotid occlusion. Pretreatment with atropine or Nomega-nitro-L-arginine-methyl-ester (L-NAME) prevented the L-KYN induced enhancement of the normal and the ischemic CCBF alike.

KYNA improved behavioral recovery within 10 days from paralysis induced by the focal PIT, as evaluated with beam walking

Pretreatment with systemic administration of KYN (i.p.) reduced infarct size (in mice) and hippocampal CA1 pyramidal cell loss, preventing post-stroke behavioral changes (hypermotility and decreased spontaneous alternation) in gerbils.

L-KYN administration decreased the number of neurons injured in the cortex, not only in the pre-treated animals, but also in those which received L-KYN after the ischemic insult.

The systemic administration (i.p.) of KYN + probenecid (only pre-treatment strategy) reduced hippocampal CA1 pyramidal cell loss and preserved LTP expression at the Schaffer collateral-CA1 synapses.

IDO is overexpressed in hippocampal CA1 area $72 \mathrm{~h}$ post-ischemia and is co-localized with Neu-N. This upregulation is possibly independent of IFN- $\gamma$.

$\mathrm{L}-\mathrm{KYN}+$ Probenecid cause neuroprotection in a global ischemic rat model. L-KYN increase the blood flow in control rabbits and ischemic animals.

The systemically administered KYNA analog (both pre and post-treatment strategies) reduced hippocampal CA1 pyramidal cell loss and preserved LTP expression at the Schaffer collateral-CA1 synapses.

IDO1 is overexpressed (immunohistochemistry) in cerebral arterioles $24 \mathrm{~h}$ post-ischemia, with increased KYN/TRP ratio. After stroke, IDO-/and 1-MT-treated animals had similar pathological and neurological scores than WT animals.
The results suggest that the $\mathrm{CCBF}$ increasing effect of L-KYN might be mediated by activation of cholinergic and nitric oxide pathways.

The results suggest that intrathecal administration of a glycine receptor antagonist may facilitate behavioral recovery during the acute phase after brain infarction.

The results suggest that L-KYN can increase the brain concentration of KYNA to neuroprotective levels, suggesting the potentia clinical usefulness of L-KYN for the prevention of neuronal loss.

The results show that even the post-event administration of L-KYN may be of therapeutic benefit in the treatment of global brain ischemia.

The results suggest that administration of KYN elevates KYNA concentration in the brain to neuroprotective levels, suggesting its potential clinical usefulness for the prevention of neuronal loss in neurodegenerative diseases.

The results show that up-regulation of IDO in hippocampal neurons after transient global ischemia occurs via INF-y-independent mechanisms.

The results demonstrate that KYN treatment minimizes neuronal cell loss in a rat model of global ischemia in which excitotoxicity seems to play a major pathophysiological role.

The results suggest that the neuroprotective effect was robust in the pretreatment, and at the time of reperfusion.

The results suggest that the expression and the activity of IDO1 increase following stroke. However, such IDO1 increased expression does not appear to affect overall outcome following acute ischemic stroke. 
TABLE 1 | Continued

\begin{tabular}{|c|c|c|c|}
\hline References & Animal model & Results & Conclusion \\
\hline Hsieh et al. (47) & $\begin{array}{l}\text { KYNA in heatstroke in adult male } \\
\text { Sprague-Dawley rats }\end{array}$ & $\begin{array}{l}\text { KYNA decreased the survival time. } \\
\text { Vehicle-treated heatstroke rats displayed } \\
\text { hypotension, hypothalamic neuronal } \\
\text { degeneration and apoptosis, increased serum } \\
\text { levels of TNF- } \alpha \text {, ICAM-1, and IL-10, and spleen, } \\
\text { liver, kidney, and lung apoptosis. KYNA } \\
\text { preconditioning protected against hypotension } \\
\text { but not hyperthermia and attenuated } \\
\text { hypothalamic neuronal degeneration and } \\
\text { apoptosis during heatstroke. KYNA } \\
\text { preconditioning attenuated spleen, kidney, liver, } \\
\text { and lung apoptosis and up-regulated serum } \\
\text { IL-10 levels but down-regulated serum TNF- } \alpha \\
\text { and ICAM-1 levels during heatstroke. }\end{array}$ & $\begin{array}{l}\text { The results suggest that systemic delivery of } \\
\text { KYNA may attenuate multi-organ dysfunction in } \\
\text { rats after heatstroke. }\end{array}$ \\
\hline
\end{tabular}

Gellert et al. (48) MCAO in adult male Wistar rats.

Cuartero et al. (49)

Lee et al. (50)

Mangas et al. (51)

Mangas et al. (52)
$\mathrm{MCAO}$ in adult male C57BL/6 mice + oxygen- glucose deprivation in rat cortical neurons

Protein levels of KYNA after ischemic preconditioning in male Mongolian gerbils

tMCAO in adult male Wistar rats

New therapeutic approach Gemst in stroke in male Wistar rat brain.
AhR is overexpressed 5 to $72 \mathrm{~h}$ post-ischemia. $\mathrm{AhR}^{-1-}$ or animals treated with $\mathrm{AhR}$ antagonists had decreased infarct size and neurological deficits. The administered L-KYN increased infarct size in an AhR-dependent manner. In vitro results confirmed that KYN is a specific $A h R$ agonist in neurons. TDO (but not IDO1 or IDO2) is overexpressed 5 to $24 \mathrm{~h}$ post-ischemia and is co-localized with Neu-N. TDO inhibitor (680C91) reduced infarct size, but not IDO1 inhibitor (1-MT).

In the ischemia-operated group, a loss of pyramidal neurons in the CA1 stratum pyramidale (SP) at 5 days post-ischemia; however, in the IPC + ischemia-operated group, the pyramidal neurons were protected. KYNA immunoreactivity in the SP of the ischemia-operated group was altered following ischemia-reperfusion and was very low 5 days following ischemia-reperfusion. In the IPC + ischemia-operated group, however, KYNA immunoreactivity was detected in the SP of the $\mathrm{CA} 1$ region after the ischemic insult. Alteration of the KYNA protein level in the CA1 region following ischemia was similar to the immunohistochemical changes observed.

KYNA is overexpressed (immunohistochemistry) in infarcted areas from day 2 to 21 post-ischemia and is co-localized with GFAP.

Gemst reversed the pathological conditions of stroke to normal situations. Gemst exerts a multifunctional action: down-regulates the indoleamine 2, 3-dioxygenase pathway and abolishes brain infiltration, microglial activation and gliosis. Moreover, Gemst has no effect on the expression of doublecortin, a protein involved in neuronal migration.
The results suggest that treatment with L-KYNs worsened the histopathological outcome of $\mathrm{dMCAO}$. This contradictory result indicates that post-ischemic treatment with L-KYNs may be harmful.

The results suggest that a L-KYN/AhR pathway mediates acute brain damage after stroke, opening new possibilities for the diagnosis and treatment.

The results indicate that the enhancement of KYNA expression by IPC may be necessary for neuronal survival following transient ischemic injury.

The results suggest that KYNA could be involved in neuroprotective, scavenger, and/or antioxidant mechanisms.

The results suggest that Gemst could be a new drug for the treatment of stroke since it reverses the pathological findings of stroke and normalizes brain tissue conditions following an ischemic insult. 
TABLE 2 | Humans studies with stroke/ischemia and kynurenine pathway.

\begin{tabular}{|c|c|c|c|}
\hline References & Study population & Results & Conclusion \\
\hline $\begin{array}{l}\text { Darlington et al. } \\
\text { (53) }\end{array}$ & $\begin{array}{l}\text { Patients with symptoms of acute } \\
\text { stroke. Performed CY scan and } \\
\text { measure kynurenines, neopterin, lipid } \\
\text { peroxidation, S100B }\end{array}$ & $\begin{array}{l}\text { KYN pathway of tryptophan metabolism is } \\
\text { activated, with an increased KYN:TRP ratio, but } \\
\text { with a highly significant decrease in the ratio of } \\
\text { 3-HAA: anthranilic acid, which was strongly } \\
\text { correlated with infarct volume. Levels of KYNA were } \\
\text { significantly raised in patients who died within } 21 \\
\text { days compared with those who survived. The } \\
\text { results suggest that increased TRP catabolism is } \\
\text { initiated before or immediately after a stroke, and is }\end{array}$ & $\begin{array}{l}\text { The results suggest that oxidative } \\
\text { tryptophan metabolism may contribute } \\
\text { to the oxidative stress and brain damage } \\
\text { following stroke. Some form of } \\
\text { anti-inflammatory intervention between } \\
\text { the rise of S100B and the activation of } \\
\text { microglia, including inhibition of the } \\
\text { kynurenine pathway, may be valuable in } \\
\text { modifying patient morbidity and mortality }\end{array}$ \\
\hline
\end{tabular}

Brouns et al. (54) Plasma concentrations of TRP and its metabolites were measured in 149 stroke patients at admission, at $24 \mathrm{~h}$, at $72 \mathrm{~h}$ and at day 7 after stroke onset.

Gold et al. (55)

Bensimon et al. (56)

Mo et al. (57)

Ormstad et al. (58)
Patients were recruited from the acute stroke. Assessments for cognition, stroke severity, and the depressive symptoms. TRP and KYN concentrations were determined by high-performance liquid chromatography.

Patients with ischemic stroke divided into high, medium, and low depressive symptom tertiles. Measure concentrations of KYN and TRP and cytokine concentrations.

A total of 81 patients with ischemic stroke and 35 normal controls were recruited. Measure, concentration of serum hsCRP, apolipoprotein A-1 and apolipoprotein $\mathrm{B}$, triglyceride, cholesterol, high density lipoprotein (HDL). TRP, KYN and KYNA.

Acute serum levels of 5-hydroxytryptamine (5-HT), TRP catabolites (TRYCATs), and competing amino acids, as well as subsequent fatigue and depression, were measured in 45 stroke patients. related to the inflammatory response and oxidative stress, with a major change in 3-HAA levels.

KYN/TRP but not KA/3-HAA correlated with the NIHSS score and with the infarct volume. Patients with poor outcome had higher mean KYN/TRP ratios than patients with more favorable outcome. The KYN/TRP ratio at admission correlated with CRP levels, ESR and NLR. The activity of the kynurenine pathway for tryptophan degradation in acute ischemic stroke correlates with stroke severity and long-term stroke outcome.

Higher KYN/TRP ratios were associated with lower post-stroke global cognition. A backward stepwise elimination linear regression showed that the highest KYN/TRP ratio tertile predicted lower SMMSE scores, controlling for age with NIHSS, and lesion volume.

No differences in KYN/TRP ratios between CES-D for cytokines ( $n=53$ ), serum $\mathrm{IL}-1 \beta$ concentrations and serum ratios of IL-18/IL-10, IFN $\gamma / \mathrm{IL}-10$, and $\mathrm{IL}-1 \beta / \mathrm{IL}-10$ were elevated in the middle CES-D tertile. Post hoc analyses suggested that serum ratios of IL-18/IL-10, and IL-1 $\beta / \mathrm{IL}-10$, as well as $\| \mathrm{L}-1 \beta$, were significantly associated with fatigue.

Lower TRP, KYNA, HDL, and KAT activity ratio were found in the stroke group compared to the control group. The levels of hsCRP and IDO activity ratio were much higher in the stroke group than the control group. The IDO activity in patients with ischemic stroke showed a positive correlation with hsCRP. In addition, hsCRP and IDO levels were positively associated with the NIHSS score both at admission and 3 weeks post-stroke.

TRP index $[=100 \times$ TRP / (tyrosine + valine + phenylalanine + leucine + isoleucine)] was lower in patients with a Fatigue Severity Scale (FSS) score of $\geq 4$ at 12 months than in those with an FSS score of $<4$. Furthermore, the serum level of KYNA in the acute stroke phase was higher in patients with an FSS of score $\geq 4$ at 18 months than in those with an FSS score of $<4$. These findings indicate that stroke patients with PSF have a lower bioavailability of TRP for 5-HT synthesis in the brain in the acute stroke phase.
The results suggest the activity of the KYN pathway for tryptophan degradation in acute ischemic stroke correlates with stroke severity and long-term stroke outcome. Tryptophan oxidation is related to the stroke induced inflammatory response.

The results suggest an inflammatory response characterized by IDO activation may be relevant to the development of PSCI. Since the neuroactivity of KYN metabolites may be amenable to pharmacotherapeutic intervention, the KYN/TRP ratio may be a clinically important biomarker.

The results suggest that peripheral $\mathrm{KYN}$ /TRP ratios were not associated with depressive symptoms in a post-stroke population. However, in exploratory analyses a pro-inflammatory bias was identified specifically in patients with mild depressive symptoms and associated with post-stroke fatigue, suggesting an avenue for future research.

The results suggest an inflammatory response characterized by up-regulated IDO activation in ischemic stroke, which might be closely relevant to its pathophysiology.

The findings indicate that stroke patients with PSF have a lower bioavailability of TRP for 5-HT synthesis in the brain in the acute stroke phase. However, they also appear to have greater neuroprotective potential in that phase. In contrast to PSF, no predictors of PSD were found. 
TABLE 3 | Main results of KYN pathway and stroke.

\begin{tabular}{|c|c|c|c|c|c|c|c|c|c|c|c|c|}
\hline \multirow[t]{2}{*}{ Specie } & \multicolumn{2}{|c|}{ KYN } & \multicolumn{2}{|c|}{ KYNA } & \multicolumn{2}{|l|}{ QA } & \multicolumn{2}{|l|}{ IDO } & \multicolumn{2}{|c|}{ TRP } & \multicolumn{2}{|l|}{ Treatments } \\
\hline & Periphery & Brain & Periphery & Brain & Periphery & Brain & Periphery & Brain & Periphery & Brain & KYNA/KYN/analog & IDO inhibitors \\
\hline Rats & NA & NA & NA & $\uparrow$ & NA & $\uparrow$ & NA & NA & NA & NA & Protection/Worsened/ no effect & Protection \\
\hline Gerbils & NA & NA & NA & No changes & NA & $\uparrow / \downarrow$ & NA & $\uparrow$ & NA & NA & Protection & Protection \\
\hline Mice & NA & NA & NA & NA & NA & NA & NA & $\uparrow$ & NA & NA & Protection & NA \\
\hline Rabbit & NA & NA & NA & NA & NA & NA & NA & NA & NA & NA & Protection & NA \\
\hline Humans & $\uparrow$ & NA & $\downarrow / \uparrow$ & NA & NA & NA & $\uparrow$ & NA & $\downarrow$ & NA & NA & NA \\
\hline
\end{tabular}

$\downarrow$ levels decreased; $\uparrow$ levels increased.

3-hydroxyanthranilic acid can generate reactive oxygen species, such as hydrogen peroxide and superoxide, in the presence of transition metal ions $(59,60)$.

Plasma levels of tryptophan and KP metabolites were measured in 149 stroke patients at admission, $24 \mathrm{~h}, 72 \mathrm{~h}$, and day 7 after stroke. Patients with poor outcome had higher kynurenine/tryptophan ratio, an index of IDO activity and KP activation, compared to patients with favorable outcomes. Moreover, the activity of IDO, as estimated by kynurenine/tryptophan ratio, correlated with stroke severity and clinical outcome (54). In line with this result, Gold et al. reported higher kynurenine/tryptophan ratio in patients with impaired post-stroke cognition as assessed by the Mini-Mental State Examination within 1 month post-stroke (55).

In the study by Bensimon et al. patients with ischemic stroke were categorized based on their depressive symptoms into high, medium, and low severity in the acute phase of the stroke. Kynurenine and tryptophan were also determined. There was no difference in kynurenine/tryptophan ratio among depressive groups (56).

\section{DISCUSSION}

There is evidence implicating the KP in the pathophysiology of stroke. Preclinical evidence also suggests that treatment with kynurenine and $\mathrm{KMO}$ inhibitors can be neuroprotective strategies to decrease infarct size and the related clinical outcomes.

In the $\mathrm{KP}$, tryptophan is degraded to kynurenine that can be converted to kynurenic acid by KATs expressed in astrocytes (3). Kynurenic acid plays neuroprotective roles in the central nervous system as a NMDA and $\alpha 7$-nicotinic acetylcholine receptor antagonist (61). Corroborating this concept, a study with intrathecal administration of kynurenic acid improved motor outcome in rats subjected to photochemically induced brain thrombosis (39). Nevertheless, peripheral administration of kynurenic acid or its agonists did not lead to similar results because its penetration through the blood-brain barrier is poor (62). Interestingly, peripheral administration of kynureninethat can cross the blood-brain barrier and then be converted to kynurenic acid in astrocytes-improved neurological outcome after stroke $(40,63,64)$. In contrast to these reports, Gellert et al. (48) showed that post-ischemic treatment of adult male rats subjected to distal middle cerebral artery occlusion for 30 min with kynurenine resulted in worsened glial reaction and neuronal death (48). The extension of brain damage in the different models and the timing of treatment could explain this contradicting finding as administered kynurenine can be converted to quinolinic acid by $\mathrm{KMO}$ expressed in activated microglia and infiltrating leukocytes in the infarct area (6). Quinolinic acid is a neurotoxic agent, acting through multiple mechanisms including NMDA receptor agonism (65). Supporting these assumptions, there is evidence from human studies showing increased levels of quinolinic acid in microglia from the brain of patients with depression (66). Interestingly enough, depressive disorders are very common post-stroke complications, affecting over $30 \%$ of patients, which corroborates the view that KP may play a role in the pathophysiology of stroke (67).

Evidence from experimental models of stroke, as presented here, neurodegenerative diseases and other neuropsychiatric conditions such as Alzheimer's disease, Huntington's disease and depression, shows that KMO inhibition prevents neuronal loss and the related cognitive and behavioral changes $(5,7)$. As the available KMO inhibitors do not cross the bloodbrain barrier and $\mathrm{KMO}$ is highly expressed in peripheral immune cells such as macrophages, it has been proposed that their peripheral action is responsible for these neuroprotective effects (68). In support of this, peripheral inhibition of KMO leads to sustained elevation in the brain levels of kynurenic acid without increasing quinolinic acid levels in the blood or brain (5). However, increased levels of kynurenic acid in the brain may not always be neuroprotective. Kindler et al. showed that patients with schizophrenia had increased kynurenic acid levels that were associated with brain volume loss and attention impairment (69). Accordingly, treatment in stroke should be aimed at "normalizing" and/or rebalancing the levels of kynurenic acid.

IDO is mainly expressed in monocytes, macrophages, dendritic cells and microglia, the tissue-resident phagocytes of the brain (70). IDO is the rate-limiting enzyme of the KP in these cells, and can be upregulated by pro-inflammatory stimuli, especially interferon-gamma (IFN- $\gamma)(71)$. Accordingly, IDO is highly expressed in infarcted brain areas. Despite that, only one study so far investigated the potential benefits of IDO inhibitors in stroke or ischemia model. The study from Jackman et al. showed that although IDO expression and activity are increased 
after transient MCAO in mice, genetic and pharmacological methods for IDO inhibition after ischemia (IDO knockout mice and 1-MT treatment, respectively) had no positive effects on infarct volume and neurological outcome, however the authors only examined the effect of IDO1 expression on outcome at $24 \mathrm{~h}$ and no later time points (46). IDO inhibitors could be a potential treatment for stroke, however more studies using IDO inhibitors in stroke are warranted to better understand of its mechanism. It is worth mentioning that inhibitors of IDO1 has being used in experiments in oncology (72). As IDO1 has the ability to establish a tumor-promoting inflammatory environment, IDO1 inhibitors decrease tumor-related inflammation and angiogenesis, potentiating the efficacy of cytotoxic or targeted chemotherapies, radiotherapy, immune checkpoint therapies and cancer vaccines (72). This strategy of decreasing the inflammation by IDO inhibitors could be explored in the treatment of stroke and related complications.

Even though several studies were concordant with the concept that KP-based treatments are potentially neuroprotective in stroke, several methodological limitations must be acknowledged. In pre-clinical studies, there were significant differences in the stroke models (focal vs. global ischemia; permanent vs. transient ischemia), in the species used (rats, Gerbils, mice, rabbits) and in the time/dose of pharmacological manipulations. Quality assessment of these studies also suggested a high risk of bias. Moreover, most studies did not take into account sex differences that have been recognized as relevant in the pathophysiology of stroke (73), while no study evaluated aged animals. In addition, there is only one study investigating $\mathrm{KP}$ in endothelial cells. Endothelial cells play a critical role in vascular homeostasis and stroke development, with damaged endothelial cells potentially triggering a series of cerebrovascular injuries (74). Finally, most pre-clinical studies only addressed neuroprotection as assessed by infarct size, with limited evaluation of behavioral measures or underlying mechanisms. More carefully designed studies controlling for age and sex

\section{REFERENCES}

1. Hankey GJ. Stroke. Lancet. (2017) 389:641-54. doi: 10.1016/S01406736(16)30962-X

2. Schwarcz R, Bruno JP, Muchowski PJ, Wu HQ. Kynurenines in the mammalian brain: when physiology meets pathology. Nat Rev Neurosci. (2012) 13:465-77. doi: 10.1038/nrn3257

3. Badawy AA. Kynurenine pathway of tryptophan metabolism: regulatory and functional aspects. Int J Tryptophan Res. (2017) 10:1178646917691938. doi: $10.1177 / 1178646917691938$

4. Kanai M, Nakamura T, Funakoshi H. Identification and characterization of novel variants of the tryptophan 2,3-dioxygenase gene: differential regulation in the mouse nervous system during development. Neurosci Res. (2009) 64:111-7. doi: 10.1016/j.neures.2009.02.004

5. Zwilling D, Huang SY, Sathyasaikumar KV, Notarangelo FM, Guidetti P, Wu HQ, et al. Kynurenine 3-monooxygenase inhibition in blood ameliorates neurodegeneration. Cell. (2011) 145:863-74. doi: 10.1016/j.cell.2011.05.020

6. Cuartero MI, de la Parra J, Garcia-Culebras A, Ballesteros I, Lizasoain I, Moro MA. The kynurenine pathway in the acute and chronic phases of cerebral ischemia. Curr Pharm Des. (2016) 22:1060-73. differences, and assessing the cells expressing the KP enzymes are definitely warranted.

Human studies corroborate the experimental findings of $\mathrm{KP}$ activation after stroke. Interestingly enough, KP-related biomarkers correlated with stroke severity and clinical outcomes, including cognitive impairment. These findings must be seen as preliminary as most studies did not include a control group and did not perform a careful behavioral, cognitive and socio-occupational phenotyping of the patients. In this regard, it would be important to specifically address which clinical parameters (i.e., neurological impairment, cognitive dysfunction, depression) are better predicted by these biomarkers. Another limitation here would be the standard method for KP metabolites assessment, i.e., liquid chromatography-mass spectrometry (LC-MS) that prevents the scaling of their measurement. In addition, only two studies compared patients with health controls.

In conclusion, $\mathrm{KP}$ is a promising target for the development of neuroprotective strategies against stroke.

\section{DATA AVAILABILITY}

All datasets analyzed for this study are included in the manuscript and/or the supplementary material.

\section{AUTHOR CONTRIBUTIONS}

GC performed the search in the literature, screening of the papers and wrote the manuscript. AT helped screening of the papers, wrote the manuscript and mentored. VV and LM reviewed the manuscript.

\section{FUNDING}

The Neuropsychiatry Program is funded by the Department of Psychiatry and Behavioral Sciences, The University of Texas Health Science Center at Houston.

7. Parrott JM, O'Connor JC. Kynurenine 3-monooxygenase: an influential mediator of neuropathology. Front Psychiatry. (2015) 6:116. doi: $10.3389 /$ fpsyt.2015.00116

8. Ying $\mathrm{W}$. NAD+/NADH and NADP+/NADPH in cellular functions and cell death: regulation and biological consequences. Antioxid Redox Signal. (2008) 10:179-206. doi: 10.1089/ars.2007.1672

9. Wang P, Miao CY. NAMPT as a therapeutic target against stroke. Trends Pharmacol Sci. (2015) 36:891-905. doi: 10.1016/j.tips.2015. 08.012

10. Huang Q, Sun M, Li M, Zhang D, Han F, Wu JC, et al. Combination of $\mathrm{NAD}(+)$ and $\mathrm{NADPH}$ offers greater neuroprotection in ischemic stroke models by relieving metabolic stress. Mol Neurobiol. (2018) 55:6063-75. doi: 10.1007/s12035-017-0809-7

11. Ao LY, Yan YY, Zhou L, Li CY, Li WT, Fang WR, et al. Immune cells after ischemic stroke onset: roles, migration, and target intervention. J Mol Neurosci. (2018) 66:342-55. doi: 10.1007/s12031-018-1173-4

12. Moher D, Liberati A, Tetzlaff J, Altman DG, Group P. Preferred reporting items for systematic reviews and meta-analyses: the PRISMA statement. PLoS Med. (2009) 6:e1000097. doi: 10.1371/journal.pmed.10 00097 
13. Hooijmans CR, Rovers MM, de Vries RB, Leenaars M, Ritskes-Hoitinga M, Langendam MW. SYRCLE's risk of bias tool for animal studies. BMC Med Res Methodol. (2014) 14:43. doi: 10.1186/1471-2288-14-43

14. Simon RP, Young RS, Stout S, Cheng J. Inhibition of excitatory neurotransmission with kynurenate reduces brain edema in neonatal anoxia. Neurosci Lett. (1986) 71:361-4.

15. Germano IM, Pitts LH, Meldrum BS, Bartkowski HM, Simon RP. Kynurenate inhibition of cell excitation decreases stroke size and deficits. Ann Neurol. (1987) 22:730-4. doi: 10.1002/ana.4102 20609

16. Andine P, Lehmann A, Ellren K, Wennberg E, Kjellmer I, Nielsen T, et al. The excitatory amino acid antagonist kynurenic acid administered after hypoxic-ischemia in neonatal rats offers neuroprotection. Neurosci Lett. (1988) 90:208-12.

17. Roussel S, Pinard E, Seylaz J. Kynurenate does not reduce infarct size after middle cerebral artery occlusion in spontaneously hypertensive rats. Brain Res. (1990) 518:353-5.

18. Heyes MP, Nowak TS Jr. Delayed increases in regional brain quinolinic acid follow transient ischemia in the gerbil. J Cereb Blood Flow Metab. (1990) 10:660-7. doi: 10.1038/jcbfm.1990.119

19. Roucher P, Meric P, Correze JL, Mispelter J, Tiffon B, Lhoste JM, et al. Metabolic effects of kynurenate during reversible forebrain ischemia studied by in vivo 31P-nuclear magnetic resonance spectroscopy. Brain Res. (1991) 550:54-60.

20. Katayama Y, Kawamata T, Kano T, Tsubokawa T. Excitatory amino acid antagonist administered via microdialysis attenuates lactate accumulation during cerebral ischemia and subsequent hippocampal damage. Brain Res. (1992) 584:329-33.

21. Lekieffre D, Ghribi O, Callebert J, Allix M, Plotkine M, Boulu RG. Inhibition of glutamate release in rat hippocampus by kynurenic acid does not protect CA1 cells from forebrain ischemia. Brain Res. (1992) 592:333-7.

22. Nozaki K, Beal MF. Neuroprotective effects of L-kynurenine on hypoxiaischemia and NMDA lesions in neonatal rats. J Cereb Blood Flow Metab. (1992) 12:400-7. doi: 10.1038/jcbfm.1992.57

23. Saito K, Nowak TS Jr, Markey SP, Heyes MP. Mechanism of delayed increases in kynurenine pathway metabolism in damaged brain regions following transient cerebral ischemia. J Neurochem. (1993) 60:180-92.

24. Saito K, Nowak TS Jr, Suyama K, Quearry BJ, Saito M, Crowley JS, et al. Kynurenine pathway enzymes in brain: responses to ischemic brain injury versus systemic immune activation. J Neurochem. (1993) 61:2061-70.

25. Saito K, Quearry BJ, Saito M, Nowak TS Jr, Markey SP, Heyes MP. Kynurenine 3-hydroxylase in brain: species activity differences and effect of gerbil cerebral ischemia. Arch Biochem Biophys. (1993) 307:104-9. doi: 10.1006/abbi.1993.1567

26. Zoli M, Merlo Pich E, Ferraguti F, Biagini G, Fuxe K, Agnati LF. Indolepyruvic acid treatment reduces damage in striatum but not in hippocampus after transient forebrain ischemia in the rat. Neurochem Int. (1993) 23:139-48.

27. Ghribi O, Callebert J, Plotkine M, Boulu RG. Effect of kynurenic acid on the ischaemia-induced accumulation of glutamate in rat striatum. NeuroReport. (1994) 5:435-7. doi: 10.1097/00001756-199401120-00016

28. Saito K, Seishima M, Noma A, Suyama K, Markey SP, Heyes MP. 4chloro-3-hydroxyanthranilate attenuate quinolinic acid accumulation in brain following transient cerebral ischemia in the gerbil. Adv Exp Med Biol. (1996) 398:407-11.

29. Heyes MP, Saito K, Chen CY, Proescholdt MG, Nowak TS Jr, Li J, et al. Species heterogeneity between gerbils and rats: quinolinate production by microglia and astrocytes and accumulations in response to ischemic brain injury and systemic immune activation. J Neurochem. (1997) 69:1519-29.

30. Baratte S, Molinari A, Veneroni O, Speciale C, Benatti L, Salvati P. Temporal and spatial changes of quinolinic acid immunoreactivity in the gerbil hippocampus following transient cerebral ischemia. Brain Res Mol Brain Res. (1998) 59:50-7.

31. Cozzi A, Carpenedo R, Moroni F. Kynurenine hydroxylase inhibitors reduce ischemic brain damage: studies with (m-nitrobenzoyl)-alanine (mNBA) and 3,4-dimethoxy-[-N-4-(nitrophenyl)thiazol-2yl]-benzenesulfonamide (Ro 61-8048) in models of focal or global brain ischemia. J Cereb Blood Flow Metab. (1999) 19:771-7. doi: 10.1097/00004647-19990700000007
32. Phillis JW, Song D, Guyot LL, O’Regan MH. Failure of kynurenic acid to inhibit amino acid release from the ischemic rat cerebral cortex. Neurosci Lett. (1999) 273:21-4.

33. Salvati P, Ukmar G, Dho L, Rosa B, Cini M, Marconi M, et al. Brain concentrations of kynurenic acid after a systemic neuroprotective dose in the gerbil model of global ischemia. Prog Neuropsychopharmacol Biol Psychiatry. (1999) 23:741-52.

34. Edwards SR, Mather LE, Lin Y, Power I, Cousins MJ. Glutamate and kynurenate in the rat central nervous system following treatments with tail ischaemia or diclofenac. J Pharm Pharmacol. (2000) 52:59-66. doi: 10.1211/0022357001773698

35. Moroni F, Cozzi A, Peruginelli F, Carpenedo R, and Pellegrini-Giampietro DE. Neuroprotective effects of kynurenine-3-hydroxylase inhibitors in models of brain ischemia. Adv Exp Med Biol. (2000) 467:199-206. doi: 10.1007/978-1-4615-4709-9_26

36. Luchowska E, Luchowski P, Sarnowska A, Wielosz M, Turski WA, Urbanska EM. Endogenous level of kynurenic acid and activities of kynurenine aminotransferases following transient global ischemia in the gerbil hippocampus. Pol J Pharmacol. (2003) 55:443-7.

37. Moroni F, Carpenedo R, Cozzi A, Meli E, Chiarugi A, Pellegrini-Giampietro DE. Studies on the neuroprotective action of kynurenine mono-oxygenase inhibitors in post-ischemic brain damage. Adv Exp Med Biol. (2003) 527:12736. doi: 10.1007/978-1-4615-0135-0_15

38. Sas K, Csete K, Vecsei L, Papp JG. Effect of systemic administration of L-kynurenine on corticocerebral blood flow under normal and ischemic conditions of the brain in conscious rabbits. J Cardiovasc Pharmacol. (2003) 42:403-9. doi: 10.1097/00005344-200309000-00012

39. Abo M, Yamauchi H, Suzuki M, Sakuma M, Urashima M. Facilitated beamwalking recovery during acute phase by kynurenic acid treatment in a rat model of photochemically induced thrombosis causing focal cerebral ischemia. Neurosignals. (2006) 15:102-10. doi: 10.1159/000094876

40. Gigler G, Szenasi G, Simo A, Levay G, Harsing LG Jr, Sas K, et al. Neuroprotective effect of L-kynurenine sulfate administered before focal cerebral ischemia in mice and global cerebral ischemia in gerbils. Eur J Pharmacol. (2007) 564:116-22. doi: 10.1016/j.ejphar.2007.02.029

41. Robotka H, Toldi J, Vécsei L. L-kynurenine: metabolism and mechanism of neuroprotection. Future Neurol. (2008) 3:169-88. doi: 10.2217/14796708.3.2.169

42. Sas K, Robotka H, Rózsa É, Ágoston M, Szénási G, Gigler G, et al. Kynurenine diminishes the ischemia-induced histological and electrophysiological deficits in the rat hippocampus. Neurobiol Dis. (2008) 32:302-8. doi: 10.1016/j.nbd.2008.07.013

43. Hoshi M, Saito K, Murakami Y, Taguchi A, Fujigaki H, Tanaka R, et al. Marked increases in hippocampal neuron indoleamine 2, 3-dioxygenase via IFNgamma-independent pathway following transient global ischemia in mouse. Neurosci Res. (2009) 63:194-8. doi: 10.1016/j.neures.2008.12.003

44. Sas K. Potential role of glutamate neurotransmission in the pathogenesis of ischemic brain damage and of depression. Effects of L-kynurenine on the survival of the hippocampal neurons and on the corticocerebral blood flow in ischemic animal models. Ideggyogy Sz. (2010) 63:61-70.

45. Gellert L, Fuzik J, Goblos A, Sarkozi K, Marosi M, Kis Z, et al. Neuroprotection with a new kynurenic acid analog in the four-vessel occlusion model of ischemia. Eur J Pharmacol. (2011) 667:182-7. doi: 10.1016/j.ejphar.2011.05.069

46. Jackman KA, Brait VH, Wang Y, Maghzal GJ, Ball HJ, McKenzie G, et al. Vascular expression, activity and function of indoleamine 2,3dioxygenase-1 following cerebral ischaemia-reperfusion in mice. Naunyn Schmiedebergs Arch Pharmacol. (2011) 383:471-81. doi: 10.1007/s00210-0110611-4

47. Hsieh YC, Chen RF, Yeh YS, Lin MT, Hsieh JH, Chen SH. Kynurenic acid attenuates multiorgan dysfunction in rats after heatstroke. Acta Pharmacol Sin. (2011) 32:167-74. doi: 10.1038/aps.2010.191

48. Gellert L, Knapp L, Nemeth K, Heredi J, Varga D, Olah G, et al. Postischemic treatment with L-kynurenine sulfate exacerbates neuronal damage after transient middle cerebral artery occlusion. Neuroscience. (2013) 247:95101. doi: 10.1016/j.neuroscience.2013.04.063

49. Cuartero MI, Ballesteros I, de la Parra J, Harkin AL, Abautret-Daly A, Sherwin E, et al. L-kynurenine/aryl hydrocarbon receptor pathway mediates 
brain damage after experimental stroke. Circulation. (2014) 130:2040-51. doi: 10.1161/circulationaha.114.011394

50. Lee JC, Tae HJ, Cho GS, Kim IH, Ahn JH, Park JH, et al. Ischemic preconditioning protects neurons from damage and maintains the immunoreactivity of kynurenic acid in the gerbil hippocampal CA1 region following transient cerebral ischemia. Int J Mol Med. (2015) 35:1537-44. doi: $10.3892 / \mathrm{ijmm} .2015 .2171$

51. Mangas A, Yajeya J, Gonzalez N, Ruiz I, Duleu S, Geffard M, et al. Overexpression of kynurenic acid in stroke: an endogenous neuroprotector? Ann Anat. (2017) 211:33-8. doi: 10.1016/j.aanat.2017.01.002

52. Mangas A, Yajeya J, Gonzalez N, Ruiz I, Pernia M, Geffard M, et al. Gemst: a taylor-made combination that reverts neuroanatomical changes in stroke. Eur J Histochem. (2017) 61:2790. doi: 10.4081/ejh.2017.2790

53. Darlington LG, Mackay GM, Forrest CM, Stoy N, George C, Stone TW. Altered kynurenine metabolism correlates with infarct volume in stroke. Eur J Neurosci. (2007) 26:2211-21. doi: 10.1111/j.1460-9568.2007.05838.x

54. Brouns R, Verkerk R, Aerts T, De Surgeloose D, Wauters A, Scharpe $\mathrm{S}$, et al. The role of tryptophan catabolism along the kynurenine pathway in acute ischemic stroke. Neurochem Res. (2010) 35:1315-22. doi: 10.1007/s11064-010-0187-2

55. Gold AB, Herrmann N, Swardfager W, Black SE, Aviv RI, Tennen $\mathrm{G}$, et al. The relationship between indoleamine 2,3-dioxygenase activity and post-stroke cognitive impairment. J Neuroinflamm. (2011) 8:17. doi: $10.1186 / 1742-2094-8-17$

56. Bensimon K, Herrmann N, Swardfager W, Yi H, Black SE, Gao FQ, et al. Kynurenine and depressive symptoms in a poststroke population. Neuropsychiatr Dis Treat. (2014) 10:1827-35. doi: 10.2147/ndt.s65740

57. Mo X, Pi L, Yang J, Xiang Z, Tang A. Serum indoleamine 2,3-dioxygenase and kynurenine aminotransferase enzyme activity in patients with ischemic stroke. J Clin Neurosci. (2014) 21:482-6. doi: 10.1016/j.jocn.2013.08.020

58. Ormstad H, Verkerk R, Amthor KF, Sandvik L. Activation of the kynurenine pathway in the acute phase of stroke and its role in fatigue and depression following stroke. J Mol Neurosci. (2014) 54:181-7. doi: 10.1007/s12031-014-0272-0

59. Goldstein LE, Leopold MC, Huang X, Atwood CS, Saunders AJ, Hartshorn M, et al. 3-Hydroxykynurenine and 3-hydroxyanthranilic acid generate hydrogen peroxide and promote alpha-crystallin cross-linking by metal ion reduction. Biochemistry. (2000) 39:7266-75. doi: 10.1021/bi992997s

60. Leipnitz G, Schumacher C, Dalcin KB, Scussiato K, Solano A, Funchal C, et al. In vitro evidence for an antioxidant role of 3-hydroxykynurenine and 3-hydroxyanthranilic acid in the brain. Neurochem Int. (2007) 50:83-94. doi: 10.1016/j.neuint.2006.04.017

61. Albuquerque EX, Schwarcz R. Kynurenic acid as an antagonist of alpha7 nicotinic acetylcholine receptors in the brain: facts and challenges. Biochem Pharmacol. (2013) 85:1027-32. doi: 10.1016/j.bcp.2012.12.014

62. Varga N, Csapo E, Majlath Z, Ilisz I, Krizbai IA, Wilhelm I, et al. Targeting of the kynurenic acid across the blood-brain barrier by core-shell nanoparticles. Eur J Pharm Sci. (2016) 86:67-74. doi: 10.1016/j.ejps.2016.02.012

63. Fukui S, Schwarcz R, Rapoport SI, Takada Y, Smith QR. Blood-brain barrier transport of kynurenines: implications for brain synthesis and metabolism. $J$ Neurochem. (1991) 56:2007-17.
64. Robotka H, Sas K, Agoston M, Rozsa E, Szenasi G, Gigler G, et al. Neuroprotection achieved in the ischaemic rat cortex with L-kynurenine sulphate. Life Sci. (2008) 82:915-9. doi: 10.1016/j.lfs.2008.02.014

65. Schwarcz R, Whetsell WO Jr, Mangano RM. Quinolinic acid: an endogenous metabolite that produces axon-sparing lesions in rat brain. Science. (1983) 219:316-8.

66. Steiner J, Walter M, Gos T, Guillemin GJ, Bernstein HG, Sarnyai Z, et al. Severe depression is associated with increased microglial quinolinic acid in subregions of the anterior cingulate gyrus: evidence for an immunemodulated glutamatergic neurotransmission? J Neuroinflammation. (2011) 8:94. doi: $10.1186 / 1742-2094-8-94$

67. Robinson RG, Jorge RE. Post-stroke depression: a review. Am J Psychiatry. (2016) 1:221-31. doi: 10.1176/appi.ajp

68. Phillips RS, Iradukunda EC, Hughes T, Bowen JP. Modulation of enzyme activity in the kynurenine pathway by kynurenine monooxygenase inhibition. Front Mol Biosci. (2019) 6:3. doi: 10.3389/fmolb.2019. 00003

69. Kindler J, Lim CK, Weickert CS, Boerrigter D, Galletly C, Liu D, et al. Dysregulation of kynurenine metabolism is related to proinflammatory cytokines, attention, and prefrontal cortex volume in schizophrenia. Mol Psychiatry. (2019). doi: 10.1038/s41380-019-0401-9. [Epub ahead of print].

70. Mandi Y, Vecsei L. The kynurenine system and immunoregulation. J Neural Transm. (2012) 119:197-209. doi: 10.1007/s00702-0110681-y

71. Lestage J, Verrier D, Palin K, Dantzer R. The enzyme indoleamine 2,3-dioxygenase is induced in the mouse brain in response to peripheral administration of lipopolysaccharide and superantigen. Brain Behav Immun. (2002) 16:596-601. doi: 10.1016/S0889-1591(02)0 0014-4

72. Prendergast GC, Malachowski WP, DuHadaway JB, Muller AJ. Discovery of IDO1 inhibitors: from bench to bedside. Cancer Res. (2017) 77:6795-811. doi: 10.1158/0008-5472.CAN-17-2285

73. Kim T, Chelluboina B, Chokkalla AK, Vemuganti R. Age and sex differences in the pathophysiology of acute CNS injury. Neurochem Int. (2019) 127:22-8. doi: 10.1016/j.neuint.2019.01.012

74. Yu QJ, Tao H, Wang $\mathrm{X}$, Li MC. Targeting brain microvascular endothelial cells: a therapeutic approach to neuroprotection against stroke. Neural Regen Res. (2015) 10:1882-91. doi: 10.4103/1673-5374.1 70324

Conflict of Interest Statement: The authors declare that the research was conducted in the absence of any commercial or financial relationships that could be construed as a potential conflict of interest.

Copyright $\odot 2019$ Colpo, Venna, McCullough and Teixeira. This is an open-access article distributed under the terms of the Creative Commons Attribution License (CC $B Y)$. The use, distribution or reproduction in other forums is permitted, provided the original author(s) and the copyright owner(s) are credited and that the original publication in this journal is cited, in accordance with accepted academic practice. No use, distribution or reproduction is permitted which does not comply with these terms. 